\title{
Metformin induces apoptosis by microRNA-26a-mediated downregulation of myeloid cell leukaemia-1 in human oral cancer cells
}

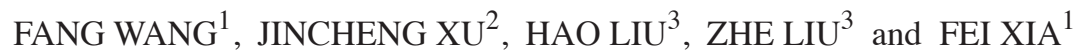 \\ ${ }^{1}$ Department of Oncology, Bengbu Medical College; ${ }^{2}$ Department of Oncology, \\ First Affiliated Hospital of Bengbu Medical College; ${ }^{3}$ Department of Biochemical Pharmacology, \\ Faculty of Pharmacy, Bengbu Medical College, Bengbu, Anhui 233000, P.R. China
}

Received August 24, 2014; Accepted May 13, 2015

DOI: $10.3892 / \mathrm{mmr} .2016 .5143$

\begin{abstract}
In recent years, population-based studies and retrospective analyses of clinical studies have shown that metformin treatment is associated with reduced cancer incidence and a decrease in cancer-associated mortality. However, its mechanism of action remains to be fully understood. The present study demonstrates the effects of metformin on KB human oral cancer cells and explores the role of myeloid cell leukaemia-1 (Mcl-1) in metformin-induced mitochondria-dependent cellular apoptosis. It was demonstrated that metformin exposure caused significant suppression of KB cell proliferation and induced cell death. Furthermore, metformin induced apoptosis through the downregulation of Mcl-1 in KB human oral cancer cells, and the overexpression of Mcl-1 in metformin-treated KB cells significantly increased cell viability. Consistently, Bax and Bim were upregulated in metformin-treated cells. The results also reveal that microRNA (miR)-26a expression was markedly increased by metformin. Subsequent to enforced miR-26a expression in KB cells using miR-26a mimics, cell viability and the level of Mcl-1 decreased. These results suggest that the anti-proliferative effects of metformin in KB cells may result partly from induction of apoptosis by miR-26a-induced downregulation of Mcl-1.
\end{abstract}

\section{Introduction}

Human oral cancer is a frequently occurring subclass of head and neck tumours. Surgery, radiation and chemotherapy are the predominant clinical therapeutic strategies for oral cancer. However, despite advances in curative multimodal treatments,

Correspondence to: Professor Jincheng $\mathrm{Xu}$, Department of Oncology, First Affiliated Hospital of Bengbu Medical College, 287 Chang Huai Road, Bengbu, Anhui 233000, P.R. China

E-mail: xjch9999@163.com

Key words: metformin, human oral cancer cells, apoptosis, Mcl-1, caspases, microRNA the overall survival rate in patients with oral cancer remains unsatisfactory $(1,2)$. Therefore, it is essential to determine the most effective therapeutic agents to control this disease and enhance patient quality of life.

Apoptosis, or programmed cell death, is regulated in a complex manner by a multitude of factors (3). B-cell lymphoma/leukaemia-2 (Bcl-2) family proteins are important regulators of cell death, and the dysregulation of Bcl-2 family members is key in certain human diseases, including cancer. Myeloid cell leukaemia-1 (Mcl-1) is a pro-survival member of the $\mathrm{Bcl}-2$ protein family that suppresses apoptosis by inhibiting the activity of pro-apoptotic proteins $(4,5)$. Moreover, Mcl-1 expression is highly amplified in a variety of human cancers and is frequently associated with chemotherapeutic resistance and recurrence, suggesting that overexpression of Mcl-1 may contribute to apoptotic evasion and malignant tumour growth. Therefore, targeting Mcl-1 expression in these cancers, using genetic and pharmacological approaches, represents a potential means of developing novel efficacious cancer therapies (6).

Metformin (1,1-dimethylbiguanide hydrochloride) is a biguanide traditional oral hypoglycaemic agent that is widely used in the clinic for the treatment of type 2 diabetes mellitus. It reduces plasma glucose levels by increasing fatty acid oxidation and glucose utilisation, decreasing hepatic gluconeogenesis (7). Recently, metformin has received considerable attention for its antitumour efficacy against numerous types of malignancies. For example, retrospective studies in patients with type 2 diabetes showed reduced cancer incidence in patients who had been treated with metformin for several years (8-11). Furthermore, other studies have demonstrated that metformin can inhibit tumour growth in vitro and increase tumour sensitivity to chemotherapeutic drugs (12-15). All of these studies strongly support the clinical development of metformin as a potentially useful therapeutic agent for cancer.

In the present study, experiments were conducted to examine the effects of metformin on KB human oral cancer cells and determine whether metformin induces apoptosis via Bcl-2 family proteins. Moreover, the present study aimed to further elucidate the mechanism underlying Mcl-1 regulation. 


\section{Materials and methods}

Chemicals and reagents. Metformin was purchased from Sigma-Aldrich (St. Louis, MO, USA). Metformin solution was prepared by dissolving metformin in phosphate-buffered saline (PBS). Minimum essential medium (MEM), foetal bovine serum (FBS) and PBS were purchased from Gibco Life Technologies (Grand Island, NY, USA). An Annexin V-fluorescein isothiocyanate (FITC)/propidium iodide (PI) apoptosis detection kit was purchased from Keygen Biotech (Nanjing, China). Anti-Mcl-1 (rabbit anti-human; 1:100), anti-Bax (rabbit anti-human; 1:1,000), anti-Bim (rabbit anti-human; 1:1,000), and anti-caspase-3 (rabbit anti-human; 1:1,000) monoclonal antibodies were obtained from Abcam (Cambridge, UK). Mouse anti- $\beta$-actin antibody $(1: 2,000)$ was obtained from Santa Cruz Biotechnology Inc. (Santa Cruz, CA, USA). Cells were transfected with miR-26a mimics, negative control or miR-26a inhibitor purchased from Guangzhou RiboBio (Guangzhou, China).

Cell lines and cell culture. The KB human oral cancer cell line was obtained from the Shanghai Cell Bank at the Chinese Academy of Sciences (Shanghai, China). Cells were cultured in MEM with $10 \%$ FBS, and supplemented with $100 \mathrm{U} / \mathrm{ml}$ penicillin and $100 \mathrm{mg} / \mathrm{ml}$ streptomycin in a humidified incubator at $37^{\circ} \mathrm{C}$ containing $5 \% \mathrm{CO}_{2}$. Cells were passaged at $80 \%$ confluence using $0.25 \%$ trypsin (Gibco Life Technologies).

Cell proliferation assay. The effect of metformin on $\mathrm{KB}$ cell viability was evaluated using the 3-(4,5-dimethylthiazolyl-2)-2-5 diphenyltetrazolium bromide (MTT) assay. KB cells in the exponential growth phase were plated in 96-well plates at $8 \times 10^{3} /$ well in a final volume of $100 \mu \mathrm{l}$. KB cells were exposed to different concentrations of metformin for 24, 48 and $72 \mathrm{~h}$ in a humidified incubator at $37^{\circ} \mathrm{C}$ with $5 \% \mathrm{CO}_{2}$. For the MTT assay, cells were then incubated with MTT $(5 \mathrm{mg} / \mathrm{ml}$ in PBS) for $4 \mathrm{~h}$. Subsequently, the MTT solution was removed and replaced with $150 \mu \mathrm{l}$ dimethyl sulphoxide/well, and the absorbance was measured at $490 \mathrm{~nm}$ using an automated microplate reader (Synergy ${ }^{\mathrm{TM}}$ HT; Bio-Tek Instruments, Inc., Winooski, VT, USA). Experiments were repeated in triplicate, and three parallel samples were measured each time.

Annexin V-FITC/PI apoptosis assay. Apoptosis was assessed using double staining with Annexin V-FITC/PI. Briefly, $\mathrm{KB}$ cells were treated with metformin at different doses in a 12-well plate for $24 \mathrm{~h}$. Cells were harvested, rinsed twice with PBS and re-suspended in $300 \mu$ ice-cold binding buffer. They were then stained with Annexin V-FITC/PI for 15 min in the dark. Stained cells were analysed using a flow cytometer (Accuri C6; BD Biosciences, Franklin Lakes, NJ, USA).

Western blot analysis. KB cells grown in 6-well plates at a density of $3 \times 10^{5}$ cells/well were treated with metformin for various incubation periods. Cells were collected by centrifugation and lysed in radioimmunoprecipitation assay buffer (Beyotime Institute of Biotechnology, Beijing, China) for $30 \mathrm{~min}$ on ice. Cell lysates were centrifuged at $12,000 \mathrm{x} \mathrm{g}$ for $30 \mathrm{~min}$ at $4^{\circ} \mathrm{C}$. Total protein $(50 \mu \mathrm{g})$ was separated by $15 \%$ sodium dodecyl sulphate-polyacrylamide gel (Beyotime
Institute of Biotechnology) electrophoresis (70 V, $30 \mathrm{~min}$; $120 \mathrm{~V}, 90 \mathrm{~min}$ ) and electrophoretically transferred to polyvinylidene fluoride membranes (Bio-Rad, Hercules, CA, USA). The membranes were blocked with 5\% non-fat dry milk for $4 \mathrm{~h}$ at room temperature and subsequently incubated with the appropriate antibody for $4 \mathrm{~h}$ at room temperature or overnight at $4^{\circ} \mathrm{C}$. After washing three times with Tris-PBS, the membranes were incubated with the corresponding secondary antibody. $\beta$-actin was used as a control for protein loading.

Plasmid transfection. The pCMV-HA-Mcl-1 plasmid and control plasmid were obtained from GenePharma (Shanghai, China). KB cells were transfected using Lipofectamine 2000 reagent (Invitrogen Life Technologies, Carlsbad, CA, USA) according to the manufacturer's instructions. After cultivation for $48 \mathrm{~h}$, total cell lysates were prepared for western blot analysis or an MTT assay.

Reverse transcription-quantitative polymerase chain reaction for miRNAs. Reverse transcription-quantitative polymerase chain reaction (RT-qPCR) was performed for miRNA detection. Total RNA was extracted from KB cells treated with or without metformin using TRIzol reagent (Invitrogen Life Technologies) and an miRNeasy Mini kit (Qiagen, Shanghai, China) according to the manufacturer's instructions. For the detection of miR-26a, mature hsa-miR-26a and U6 primer from All-in-One ${ }^{\mathrm{TM}}$ miRNA qPCR Primer (GeneCopoeia, Guangzhou, China) was used. RT-qPCR was performed with the All-in-One ${ }^{\mathrm{TM}}$ miRNA qRT-PCR Detection kit (AOMD-Q050, GeneCopoeia) in an ABI StepOne ${ }^{\mathrm{TM}}$ RealTime PCR System (Applied Biosystems Life Technologies, Foster City, CA, USA) at $95^{\circ} \mathrm{C}$ for $10 \mathrm{~min}$, followed by 40 cycles of $95^{\circ} \mathrm{C}$ for $10 \mathrm{sec}, 60^{\circ} \mathrm{C}$ for $30 \mathrm{sec}$ and $72^{\circ} \mathrm{C}$ for $15 \mathrm{sec}$. PCR data were analysed using the $2^{-\Delta \Delta C t}$ method and were normalised against RNU6B expression in each sample. Paired Student's t-test was performed to appraise the difference in the level of miRNA expression.

Transfection of miRNA precursor miR-26a. KB cells were seeded in 6-well plates at a density of $3 \times 10^{5}$ cells/well. Cells at $60-80 \%$ confluency were transfected with microRNA (miR)-26a mimics, negative control, miR-26a inhibitor, or inhibitor control at a final concentration of $20 \mu \mathrm{M}$ using Lipofectamine 2000 reagent (Invitrogen Life Technologies, Carlsbad, CA, USA) according to the manufacturer instructions. Cells were harvested $48 \mathrm{~h}$ later for western blot analysis and PI viability assays.

Plate clone formation assay. Cells were seeded at a density of $1 \times 10^{4}$ cells/well in 6-well plates with growth medium $48 \mathrm{~h}$ after transfection and incubated in MEM containing 10\% FBS at $37^{\circ} \mathrm{C}$ with $5 \% \mathrm{CO}_{2}$ for 7 days. The colonies were washed with PBS, fixed with $10 \%$ formaldehyde for $10 \mathrm{~min}$ on ice, and stained with $1.0 \%$ crystal violet for $30 \mathrm{~min}$.

Propidium iodide staining. Prior to metformin treatment, cells were plated in 12-well plates and subsequently transfected with miR-26a mimics, negative control, miR-26a inhibitor, or inhibitor control for $48 \mathrm{~h}$. Cells were then subjected to PI staining and evaluated using flow cytometry (Accuri C6). 


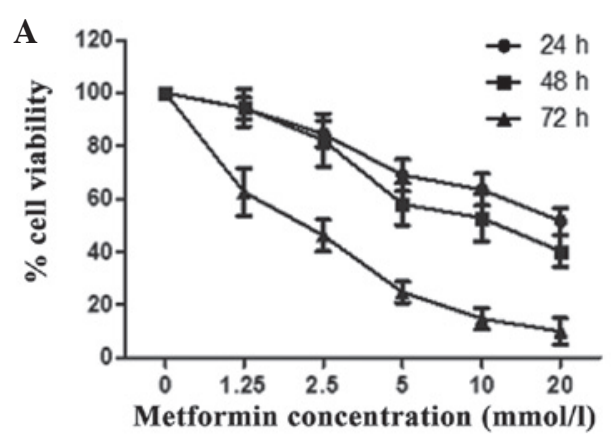

B

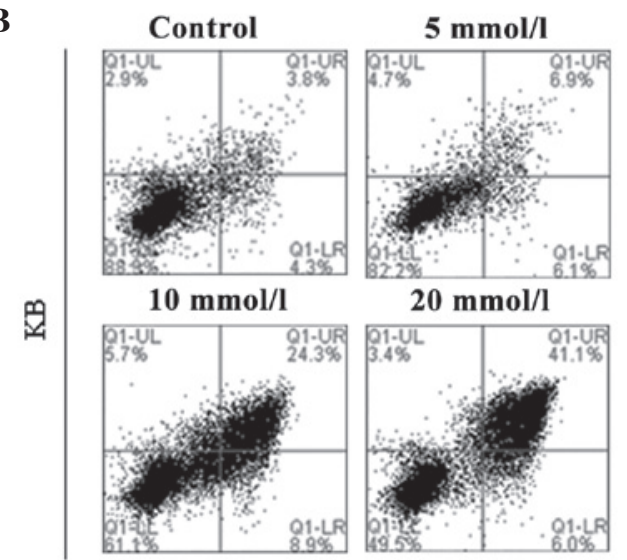

C

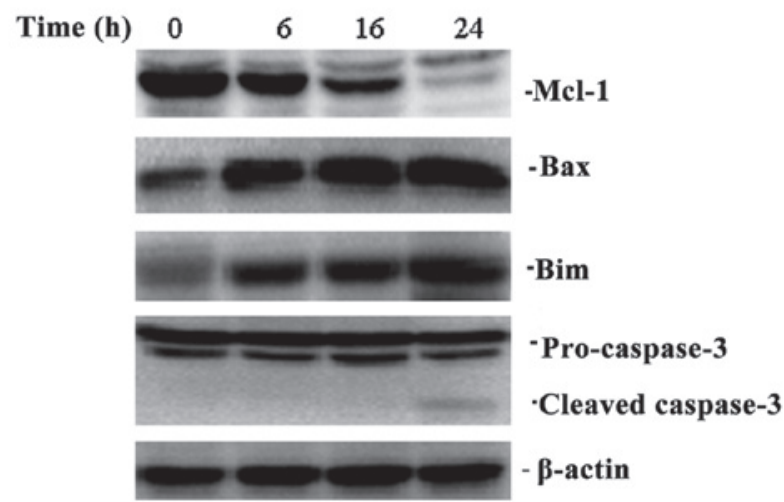

Figure 1. Metformin inhibited the proliferation and induced apoptosis of KB cells. (A) KB cells were treated with different concentrations of metformin $(0,1.25,2.5,5,10$ and $20 \mathrm{mmol} / \mathrm{l})$ for 24,48 and $72 \mathrm{~h}$, and the cell viability was analysed using an 3-(4,5-dimethylthiazolyl-2)-2-5 diphenyltetrazolium bromide assay. (B) Cells were treated with various concentrations of metformin $(5,10,20 \mathrm{mmol} / \mathrm{l})$ and were harvested after incubation for $24 \mathrm{~h}$ before being analysed using Annexin V/PI staining. (C) Whole cell lysates from KB treated with the combination of metformin $(10 \mathrm{mM})$ for $0,6,16$ and $24 \mathrm{~h}$ were subjected to western blot analysis for Mcl-1, Bax, Bim and caspase-3. One representative blot out of three is shown.

Statistical analysis. All experiments were repeated at least three times and the values were expressed as the mean \pm standard deviation. Statistical significance was determined by Student's t-test using SPSS software, version 13.0 (SPSS, Inc., Chicago, IL, USA). $\mathrm{P}<0.05$ was considered to indicate a statistically significant difference.

\section{Results}

Metformin inhibits the proliferation and induces apoptosis of $K B$ cells. To evaluate the growth inhibitory effect of metformin

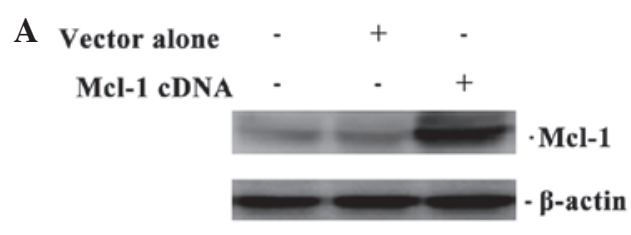

B

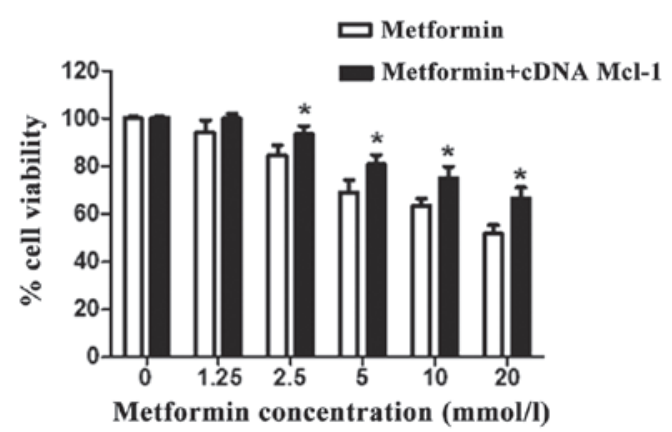

C

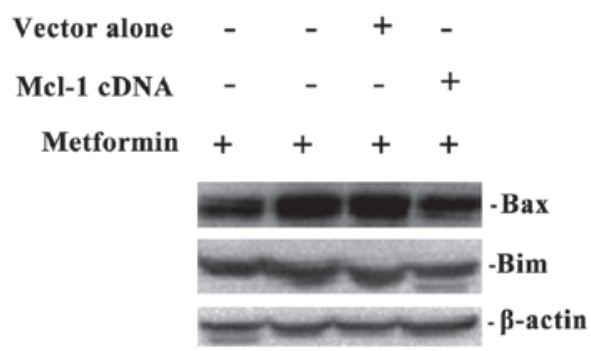

D

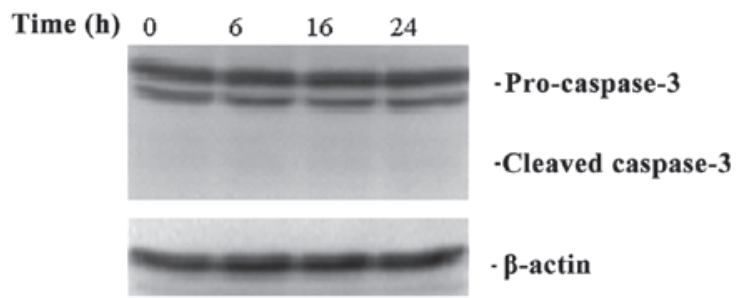

Figure 2. Upregulation of Mcl-1 protects KB cells from apoptosis. (A) Whole cell lysates from KB transfected with Mcl-1 cDNA for $48 \mathrm{~h}$ were subjected to western blot analysis. ${ }^{*} \mathrm{P}<0.05$ compared with the respective controls. (B) KB cells were transfected with Mcl-1 cDNA. After $24 \mathrm{~h}$, the cells were cultured with metformin for $24 \mathrm{~h}$, and the cell viability was analysed using an 3-(4,5-dimethylthiazolyl-2)-2-5 diphenyltetrazolium bromide assay. (C and D) KB cells were transfected with the control or Mcl-1 cDNA. After $48 \mathrm{~h}$, the total cell lysates were prepared, and western blotting was conducted. The membranes were probed with anti-Bax, -Bim and -caspase-3 antibodies. The data are representative of three independent experiments.

on human KB cells in vitro, cells were treated with different concentrations of metformin for 24,48 and $72 \mathrm{~h}$. The rate of cell proliferation was inversely related to the time of exposure to metformin and the metformin concentration (Fig. 1A).

Flow cytometric analysis revealed that metformin markedly increased the number of apoptotic KB cells (Fig. 1B). Furthermore, in KB cells, metformin decreased the expression of Mcl-1 and increased the expression of Bim and Bax in a time-dependent manner (Fig. 1C). Downstream of apoptosis signalling pathways, there was a significant activation of caspase-3 cleavage (Fig. 1C). These results confirmed the prediction that metformin induces apoptosis, and that this is predominantly through the mitochondria-mediated internal pathway. 


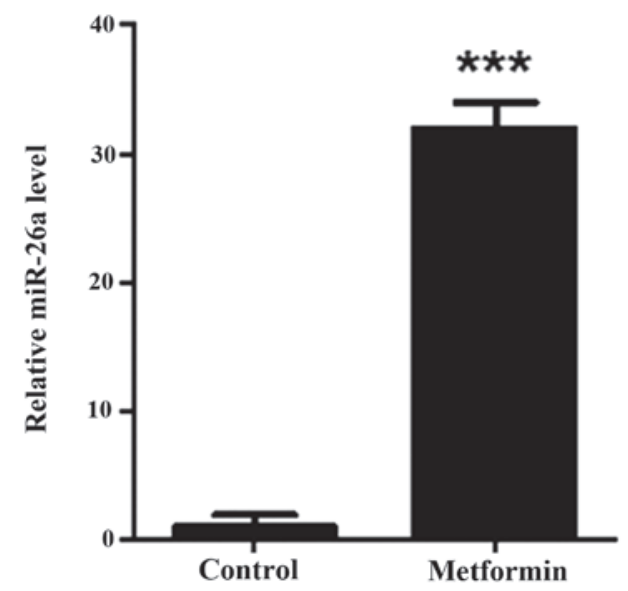

Figure 3. Metformin significantly increases the expression of miR-26a. KB cells were treated with metformin, the levels of miR-26a were measured by reverse transcription-quantitative polymerase chain reaction. ${ }^{* * *} \mathrm{P}<0.001$ compared with control.

A

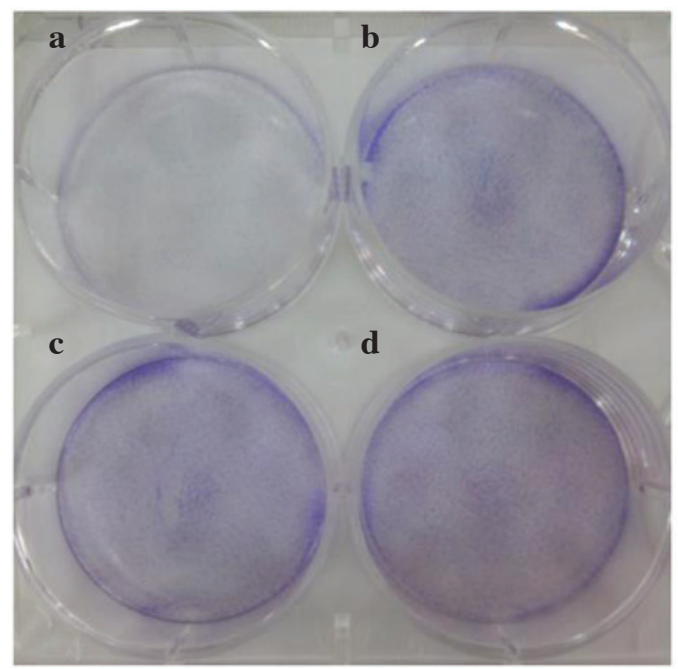

B

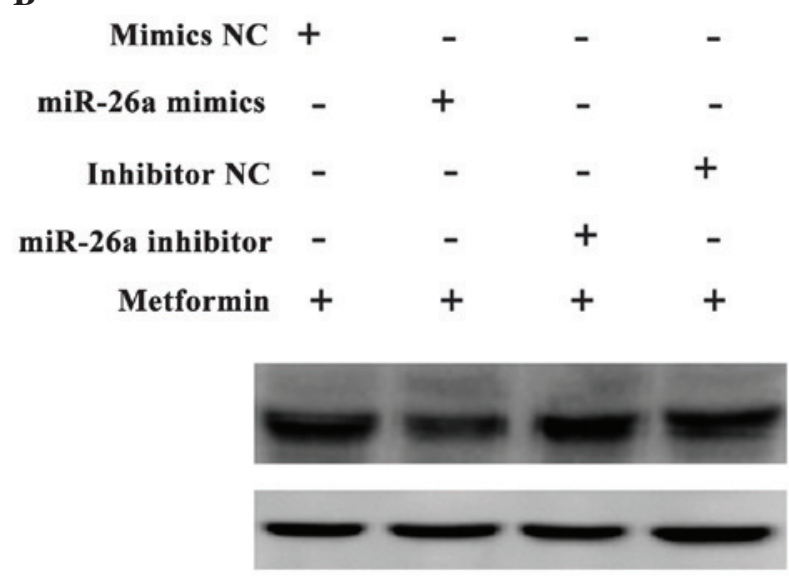

C

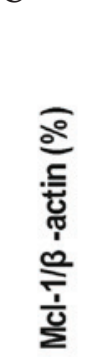

mimics NC miR-26a mimics inhibitor NC miR-26a inhibitor metformin

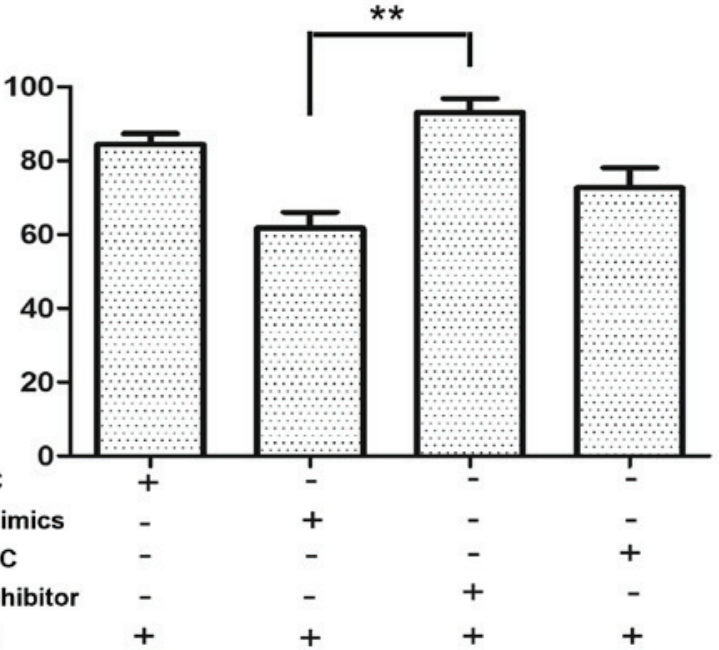

Figure 4. Overexpression of miR-26a reduced cell viability and regulated Mcl-1 expression in KB cells. miR-26a decreased KB oral cancer cell colony formation. Representative images are shown. (Aa) miR-26a mimics+metformin; (Ab) NC+metformin; (Ac) miR-26a inhibitor+metformin; (Ad) inhibitor NC+metformin. (B) Western blotting and (C) quantification of Mcl-1 expression in KB cells after transfection with miR-26a mimics and negative control. Mcl-1 expression was downregulated after KB cell transfection with miR-26a mimics. ${ }^{* *} \mathrm{P}<0.01$ compared with the combination of miR-26a mimics and metformin

Upregulation of Mcl-1 protects KB cells from apoptosis. To evaluate the association between Mcl-1 and metformin-induced apoptosis, an Mcl-1 expression plasmid was transfected into
KB cells (Fig. 2A). Then, overexpression of Mcl-1 significantly increased cell viability in response to metformin treatment and decreased the expression of Bim and Bax compared 
with their respective controls (Fig. 2B and C). In addition, the cleaved products of caspase- 3 were scarcely activated in the Mcl-1-overexpressing KB cells (Fig. 2D). These findings suggest that Mcl-1 may be important in metformin-induced apoptosis as an anti-apoptotic protein.

Metformin increases the expression of miR-26a. Expression of miR-26a was analysed by RT-qPCR and normalised against an endogenous control (RNU6B). Total RNA was extracted from $\mathrm{KB}$ cells treated with or without $10 \mathrm{mmol} / 1 \mathrm{metformin}$. The results indicated that miR-26a was significantly upregulated in metformin-treated cells compared with non-treated cells (Fig. 3).

Overexpression of miR-26a reduces cell viability and regulated Mcl-1 expression in $\mathrm{KB}$ cells. In order to confirm the biological function of miR-26a, KB cells were transfected with miR-26a mimics or corresponding negative controls for 7 days, miR-26a inhibited KB cell growth compared with miR-Ctrl (Fig. 4A). As shown in Fig. 4B, the level of Mcl-1 decreased in cells transfected with miR-26a mimics compared with cells transfected with the corresponding negative control. By contrast, Mcl-1 was increased in KB cells transfected with anti-miR-26a inhibitor compared with cells transfected with the corresponding negative control. These experiments confirm that the expression of Mcl-1 can be regulated by miR-26a.

\section{Discussion}

Metformin is widely used for the treatment of diabetes mellitus, however, recent epidemiological and preclinical studies have demonstrated that metformin is also a promising anticancer agent. In this study, exposure of KB oral cancer cells to metformin led to a time- and concentration-dependent inhibition of proliferation. Moreover, metformin was found to induce apoptosis.

Apoptosis is an activated cellular death process that is induced by physiological or pathological factors to eliminate redundant and damaged cells. Furthermore, apoptosis is an important defence against cancer (16). When cells are exposed to various stress stimuli, Bim relays apoptotic signals to the mitochondria through the activation of Bax, resulting in an increase in mitochondrial outer membrane permeabilisation and the consequent release of cytochrome $c$ into the cytosol. Cytochrome $c$ then binds to Apaf-1 to ensure the formation of the apoptosome that leads to activation of caspase- 9 and the induction of the apoptosis-promoting caspase cascade (17). By contrast, anti-apoptotic Bcl-2 proteins, such as Mcl-1 serve to inhibit apoptosis. Generally, promotion of cell survival by Mcl-1 is hypothesised to be due to sequestration of Bim, thereby inhibiting Bax/Bim interaction in the mitochondrial outer membrane. Alternatively, Mcl-1 may directly bind to Bax and maintain it in an inactive conformation. This competition leads to the suppression of cytochrome $c$ release from the mitochondria and a reduction in Apaf-1-dependent activation of caspase-3. Therefore, Mcl-1 acts in a significant regulatory role that can modulate the expression of pro-apoptotic proteins and control cell fate decisions (18). Consistent with this theory, the results of the present study demonstrate that metformin downregulated the expression of Mcl-1 and upregulated the expression of Bim and Bax. Conversely, overexpression of Mcl-1 downregulated Bim and Bax expression, suggesting that Mcl-1 is involved in $\mathrm{KB}$ cell apoptosis, and predominantly through mitochondria-mediated pathways.

In vitro and in vivo studies have shown that the expression of various miRNAs was markedly altered by treatment with metformin (19). By binding to the 3'-untranslated region (UTR) of target mRNAs, miRNAs act as endogenous sequence-specific suppressors that regulate gene expression by eliciting mRNA degradation or inhibition of mRNA translation (20). Therefore, miRNAs are important regulators of tumourigenicity, proliferation, apoptosis, invasion and metastasis. Numerous studies have highlighted the role of miRNAs in Mcl-1 regulation (21-23). Analysis of candidate target genes for miR-26a using miRBase previously revealed perfect complementarity between miR-26a and the 3' UTR of Mcl-1 over the first 9 nucleotides $(21,23)$. In the current study, it was demonstrated that miR-26a significantly downregulated Mcl-1, which led to the apoptosis of KB cells. Furthermore, miR-26a overexpression suppressed in vitro cell proliferation. Conversely, downregulation of miR-26a inhibited apoptosis and promoted proliferation. These results indicate that miR-26a may be a novel tumour suppressor that is important in the regulation of tumoural Mcl-1 expression.

In conclusion, the results of this study confirm that metformin restricted tumour growth and induced apoptosis predominantly by regulating the expression of Bcl-2 family members in the KB human oral cancer cell line. Furthermore, it was demonstrated that metformin increased expression of miR-26a in KB cells and miR-26a itself mediated inhibition of proliferation and induction of apoptosis. In combination, these findings suggest that metformin induces apoptosis in human oral cancer cells by downregulating Mcl-1 via miR-26a and these results provide in vitro evidence to support the use of metformin as a novel and efficient candidate for the treatment of human oral cancer.

\section{Acknowledgements}

This study was supported by the National Natural Science Foundation of China (grant nos. 81000992 and 81072207); the Natural Science Foundation of Anhui Province (grant no. KJ2012A201); and Graduate Scientific Research and Innovation Projects of Bengbu Medical College of the Anhui Province (grant no. Byycx1315).

\section{References}

1. Cruz GD, Ostroff JS, Kumar JV and Gajendra S: Preventing and detecting oral cancer. Oral health care providers' readiness to provide health behavior counseling and oral cancer examinations. J Am Dent Assoc 136: 594-601, 2005.

2. Rogers SN, Brown JS, Woolgar JA, Lowe D, Magennis P, Shaw RJ and Vaughan D: Survival following primary surgery for oral cancer. Oral Oncol 45: 201-211, 2009.

3. Plati J, Bucur O and Khosravi-Far R: Apoptotic cell signaling in cancer progression and therapy. Integr Biol (Camb) 3: 279-296, 2011.

4. Perciavalle RM and Opferman JT: Delving deeper: MCL-1's contributions to normal and cancer biology. Trends Cell Biol 23: 22-29, 2013.

5. Thomas LW, Lam C and Edwards SW: Mcl-1; the molecular regulation of protein function. FEBS Lett 584: 2981-2989, 2010. 
6. Quinn BA, Dash R, Azab B, Sarkar S, Das SK, Kumar S, Oyesanya RA, Dasgupta S, Dent P, Grant S, et al: Targeting Mcl-1 for the therapy of cancer. Expert Opin Investig drugs 20: 1397-1411, 2011.

7. Foretz M and Viollet B: New promises for metformin: Advances in the understanding of its mechanisms of action. Med Sci (Paris) 30: 82-92, 2014 (In French).

8. Evans JM, Donnelly LA, Emslie Smith AM, Alessi DR and Morris AD: Metformin and reduced risk of cancer in diabetic patients. BMJ 330: 1304-1305, 2005.

9. Noto H, Goto A, Tsujimoto T and Noda M: Cancer risk in diabetic patients treated with metformin: A systematic review and meta-analysis. PLoS One 7: e33411, 2012.

10. Decensi A, Puntoni M, Goodwin P, Cazzaniga M, Gennari A, Bonanni B and Gandini S: Metformin and cancer risk in diabetic patients: A systematic review and meta-analysis. Cancer Prev Res (Phila) 3: 1451-1461, 2010

11. Landman GW, Kleefstra N, van Haterenm KJ, Groenier KH Gans RO and Bilo HJ: Metformin associated with lower cancer mortality in type 2 diabetes: ZODIAC-16. Diabetes Care 33: $322-326,2010$

12. Wang F, Xu J, Xia F, Liu Z, Zhao S, Liu H and Jiang Z: Effects of metformin on human oral cancer KB cell proliferation and apoptosis in vitro. Nan Fang Yi Ke Da Xue Xue Bao 34: 159-163, 2014 (In Chinese).

13. Marini C, Salani B, Massollo M, Amaro A, Esposito AI Orengo AM, Capitanio S, Emionite L, Riondato M, Bottoni G, et al Direct inhibition of hexokinase activity by metformin at leas partially impairs glucose metabolism and tumor growth in experimental breast cancer. Cell Cycle 12: 3490-3499, 2013

14. Mohammed A, Janakiram NB, Brewer M, Ritchie RL, Marya A, Lightfoot S, Steele VE and Rao CV: Antidiabetic drug metformin prevents progression of pancreatic cancer by targeting in part cancer stem cells and mTOR signaling. Transl Oncol 6: 649-659, 2013.
15. Miyoshi H, Kato K, Iwama H, Maeda E, Sakamoto T, Fujita K, Toyota Y, Tani J, Nomura T, Mimura S, et al: Effect of the anti-diabetic drug metformin in hepatocellular carcinoma in vitro and in vivo. Int J Oncol 45: 322-332, 2014.

16. Melet A, Song K, Bucur O, Jagani Z, Grassian AR and Khosravi-Far R: Apoptotic pathways in tumor progression and therapy. Adv Exp Med Biol 615: 47-79, 2008.

17. Del Gaizo Moore V and Letai A: $\mathrm{BH} 3$ profiling-measuring integrated function of the mitochondrial apoptotic pathway to predict cell fate decisions. Cancer Lett 332: 202-205, 2013.

18. Karnak D and $\mathrm{Xu} \mathrm{L}$ : Chemosensitizaition of prostate cancer by modulating Bcl-2 family proteins. Curr Drug Targets 11: 699-707, 2010.

19. Li W, Yuan Y, Huang L, Qiao M and Zhang Y: Metformin alters the expression profiles of microRNAs in human pancreatic cancer cells. Diabetes Res Clin Pract 96: 187-195, 2012.

20. O'Hara SP, Mott JL, Splinter PL, Gores GJ and LaRusso NF: MicroRNAs: Key modulators of posttranscriptional gene expression. Gastroenterology 136: 17-25, 2009

21. Yang X, Liang L, Zhang XF, Jia HL, Qin Y, Zhu XC, Gao XM, Qiao P, Zheng Y, Sheng YY, et al: MicroRNA-26a suppresses tumor growth and metastasis of human hepatocellular carcinoma by targeting interleukin-6-Stat3 pathway. Hepatology 58: $158-170,2013$.

22. Zhang YK, Wang H, Leng Y, Li ZL, Yang YF, Xiao FJ, Li QF, Chen XQ and Wang LS: Overexpression of microRNA-29b induces apoptosis of multiple myeloma cells through down regulating Mcl-1. Biochem Biophys Res Commun 414: 233-239, 2011.

23. Gao J, Li L, Wu M, Liu M, Xie X, Guo J, Tang H and Xie X: MiR-26a inhibits proliferation and migration of breast cancer through repression of MCL-1. PLoS One 8: e65138, 2013. 